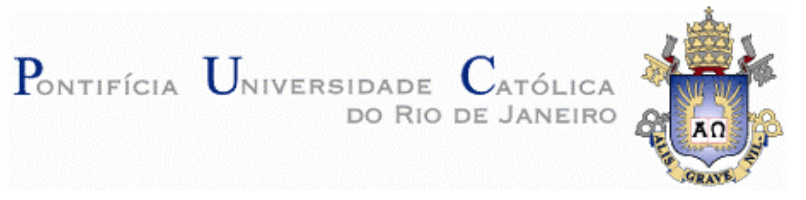

Bianca Gonçalves Moreira

\title{
Diferenciação de Bebidas Alcoólicas e Não-alcoólicas no Sistema de Medição de Vazão (SMV) durante o Envase
}

\section{Dissertação de Mestrado}

Dissertação apresentada como requisito parcial para obtenção do título de Mestre pelo Programa de PósGraduação em Metrologia da PUC-Rio. Área de Concentração: Metrologia para Qualidade e Inovação.

Professores Orientadores:

Paula Medeiros Proença de Gouvêa, Ph.D.

PósMQI/PUC-Rio

Hortencio Alves Borges, Ph.D. Departamento de Física/PUC-Rio

Rio de Janeiro

Rio de Janeiro, 19 de setembro de 2005 
Bianca Gonçalves Moreira

Diferenciação de Bebidas Alcoólicas e Não-alcoólicas no Sistema de Medição de Vazão (SMV) durante o Envase

Dissertação apresentada como requisito parcial para obtenção do grau de Mestre pelo Programa de Pós-Graduação em Metrologia, Qualidade e Inovação (PósMQI) do Centro Técnico Científico da PUC-Rio. Aprovada pela Comissão Examinadora e homologada pela Coordenação Setorial de PósGraduação, formalizado pelas respectivas assinaturas:

Comissão Examinadora:

Profa. Dra. Paula Medeiros Proença Gouvêa

Orientadora

PósMQI - PUC-Rio

Prof. Dr. Hortencio Alves Borges

Co-orientador

Departamento de Física - PUC-Rio

Dr. Luiz Carlos Guedes Valente

Gavea Sensors - Sistemas de Medição LTDA

M.Sc. Valter Yoshihiko Aibe

Inmetro

Profa. Dra. Danays Morejón González

PósMQI - PUC-Rio

Coordenação Setorial de Pós-Graduação:

Prof. José Eugênio Leal Coordenador Setorial de Pós-Graduação do Centro Técnico Científico (PUC-Rio)

Rio de Janeiro, 19 de setembro de 2005 
Todos os direitos reservados. É proibida a reprodução total ou parcial do trabalho sem autorização da universidade, da autora e dos orientadores.

\section{Bianca Gonçalves Moreira}

Graduada em Química Tecnológica pela UNICAMP Universidade Estadual de Campinas, em 1995. Química Júnior do Laboratório Central da Companhia Cervejaria Brahma de 1995 a 2001. Química Pleno do Laboratório Central da AmBev - Companhia de Bebidas das Américas, de 2001 a 2003. Especialista em Metrologia do Laboratório Central da AmBev - Companhia de Bebidas das Américas, a partir de fevereiro de 2003.

Ficha Catalográfica

Moreira, Bianca Gonçalves

Diferenciação de bebidas alcoólicas e não-alcoólicas no sistema de medição de vazão (SMV) durante o envase. I Bianca Gonçalves Moreira ; orientadores: Paula Medeiros Proença de Gouvêa, Hortencio Alves Borges. - Rio de Janeiro : PUC, Departamento de Metrologia, 2005.

143 f. ; il. ; $30 \mathrm{~cm}$

Dissertação (mestrado) - Pontifícia Universidade Católica do Rio de Janeiro, Departamento de Metrologia.

Inclui referências bibliográficas.

1. Metrologia - Teses. 2. Medição. 3. Distinção de bebidas. 4. Sistema de medição de vazão (SMV). 5. Condutivímetro. 6. Condutividade elétrica. 7. Sensor à fibra óptica. 8. Índice de refração. 9. Enchedora de bebidas. 10. Indústria de bebidas. I. Gouvêa, Paula Medeiros Proença de. II. Borges, Hortencio Alves. III. Pontifícia Universidade Católica do Rio de Janeiro. Departamento de Metrologia. IV. Título. 
Dedico este trabalho, com amor, ao meu marido Paulo, ao meu irmão Vinícius, aos meus pais Sérgio e Laura e aos queridos José e Ondina. 


\section{Agradecimentos}

À Professora Paula Gouvêa pela orientação e dedicação que contribuíram não só para o desenvolvimento deste trabalho, como também para meu aperfeiçoamento profissional.

Ao Professor Hortencio Borges pela orientação e apoio que viabilizaram a realização deste trabalho.

Ao Valter Aibe (Inmetro) pela atenção, apoio, valiosas sugestões e cooperação na redefinição do tema da dissertação, especialmente pela sugestão de testar o sensor à fibra de índice de refração da Gavea Sensors.

Ao Professor Luiz Carlos Guedes Valente que, juntamente com a equipe Gavea Sensors - Sistemas de Medição LTDA, empresa encubada do Instituto Gênesis (incubadora tecnológica da PUC-Rio) e do Laboratório de Sensores a Fibra Óptica (LSFO) do Departamento de Engenharia Mecânica da PUC-Rio, forneceu toda a infraestrutura necessária para a realização das análises utilizando o sensor de índice de refração.

À AmBev por colaborar para o desenvolvimento desta pesquisa de mestrado, que contribuiu para aprimoramento do Sistema de Medição de Vazão, liberando-me em regime de tempo parcial.

À amiga Giselle Perez, gerente do Laboratório Central da AmBev, pelo grande apoio, fundamental para o término deste trabalho, e incentivo ao meu crescimento profissional.

Ao Roger Alves (AmBev) pela atenção e apoio fundamentais na fase inicial deste trabalho.

Ao Luís Gustavo de Oliveira (AmBev) pela ajuda na realização das análises em laboratório. 
À amiga Alessandra Maia por todo incentivo e apoio para a realização do Mestrado no PósMQI.

Aos amigos do PósMQ Elizete Rangel, Carlos Eduardo Chaves, Índia Maria, Willians Portella, Luiz Alberto Lima e Fabio Zander pela amizade e parceria durante o curso.

Ao Programa Tecnologia Industrial Básica (TIB) e Serviços Tecnológicos para a Inovação e Competitividade MCT/FINEP/FNDCT/Fundo Verde Amarelo, um programa cooperativo universidade-empresa, que através do Convênio referência FINEP $n^{\circ}$ 22.01.0692.00, financiou parte do projeto. 


\section{Resumo}

Moreira, Bianca Gonçalves. Diferenciação de Bebidas Alcoólicas e Nãoalcoólicas no Sistema de Medição de Vazão (SMV) durante o Envase. Rio de Janeiro, 2005. 143p. Dissertação de Mestrado - Programa de PósGraduação em Metrologia, Qualidade e Inovação (PósMQI), Pontifícia Universidade Católica do Rio de Janeiro.

De acordo com os cálculos do Sindicato Nacional da Indústria de Cerveja (Sindicerv), os estados e o governo federal perdiam anualmente cerca de R\$ 720 milhões em arrecadação, devido à sonegação praticada por alguns fabricantes de cerveja. Um elemento facilitador para esse tipo de sonegação é o método atualmente utilizado pela Receita Federal para o controle da quantidade de volume produzido, que envolve apenas o controle de notas fiscais de vendas, o que tem sido uma grande fonte de erros e prejuízo, tanto para as empresas, quanto para o governo. Em 2001, com o objetivo de criar mecanismos que auxiliem no controle de arrecadação de impostos, o governo federal publicou a Medida Provisória $\mathrm{n}^{\mathrm{o}}$ 2.158-35, obrigando os fabricantes a instalar um Sistema de Medição de Vazão (SMV) em todas as linhas de engarrafamento, na entrada de cada enchedora. O SMV seria usado inicialmente apenas no monitoramento. Além de medir a quantidade de bebida produzida, o SMV é, por meio de um sistema de condutivímetro elétrico, responsável por detectar o tipo de líquido que está sendo envasado. Nesta dissertação, são avaliadas possíveis técnicas de diferenciação de bebidas alcoólicas e não-alcoólicas, visando atribuir maior confiança à Receita Federal e aos consumidores. Além da condutividade, foram avaliadas a densimetria e a potenciometria. Devido a problemas encontrados nestas três (3) técnicas, resolveu-se testar a eficiência de um sensor à fibra óptica de índice de refração para a diferenciação de bebidas. A principal vantagem deste sensor é tornar a sonegação mais difícil, pois, ao contrário do condutivímetro, não sofre influência eletromagnética externa. As incertezas de medição dos valores obtidos para condutividade e índice de refração foram calculadas. Os resultados da presente dissertação são suficientemente significativos para substanciar a recomendação de uma revisão da Medida Provisória $n^{0}$ 2.158-35. Parte desta 
dissertação foi financiada, através do Convênio referência FINEP $n^{\circ}$ 22.01.0692.00, pelo Programa Tecnologia Industrial Básica e Serviços Tecnológicos para a Inovação e Competitividade MCT/FINEP/FNDCT/Fundo Verde Amarelo, um programa cooperativo universidade-empresa.

\section{Palavras-chave}

Metrologia; medição; distinção de bebidas; sistema de medição de vazão (SMV); condutivímetro; condutividade elétrica; sensor à fibra óptica; índice de refração; enchedora de bebidas; indústria de bebidas. 


\section{Abstract}

Moreira, Bianca Gonçalves. Distinguishing Between Alcoholic and Non Alcoholic Beverages in the Flow Measurement System During Filling Rio de Janeiro, 2005. 143p. MSc. Dissertation - Programa de PósGraduação em Metrologia, Qualidade e Inovação (PósMQI), Pontifícia Universidade Católica do Rio de Janeiro.

According to the Brazilian Sindicato Nacional da Indústria de Cerveja (Sindicerv), about R $\$ 720$ million a year used to be lost due to federal and state tax evasion by some beer manufacturers. Tax evasion used to be eased by the method used by the Brazilian federal revenue service (Receita Federal) to monitor the amount of beer manufactured, which was solely based on sales receipts, resulting in large losses for the government and companies. In 2001, in order to create mechanisms to help in tax collection, the Federal government issued the Medida Provisória no 2.158-35, making it mandatory for the manufacturers to install a Flow Measurement System in all bottling lines, at the beginning of the beverage filler. The system was to be initially used as a monitoring device. In addition to measuring the amount of beverage manufactured, the Flow Measurement System is also responsible for detecting the type of beverage being bottled or canned by means of an electrical conductivity sensor. This and other possible methods for distinguishing between alcoholic and non alcoholic beverages in the Flow Measurement System are evaluated in this dissertation, in order to increase reliability for the Receita Federal and the consumers. In addition to the conductivity, density and $\mathrm{pH}$ measurements were also evaluated. Due to problems found in these three (3) techniques, an index of refraction optical fiber sensor was also tested for distinguishing between beverages. The main advantage of this sensor is that it makes tax evasion more difficult because, as opposed to the conductivity sensor, it is not affected by external electromagnetic interference. The uncertainty in measurement of the conductivity and index of refraction results were calculated. This dissertation's results are sufficiently significant to substantiate the recommendation that the Medida Provisória no 2.158-35 be revised. Part of this dissertation was financed by the Convênio referência FINEP $n^{\circ} 22.01 .0692 .00$ of the Programa Tecnologia Industrial Básica e Serviços Tecnológicos para a Inovação e Competitividade MCT/FINEP/FNDCT/Fundo Verde Amarelo, a university-company cooperative program. 


\section{Key-Words}

Metrology; measurement; distinguishing beverages; flow measurement system; conductivity sensor; electrical conductivity; optical fiber sensor; index of refraction; refractive index; beverage filler; beverage industry. 


\section{Sumário}

Capítulo 2 Legislação publicada sobre o SMV, participação do Inmetro e avaliação do impacto econômico 29

2.1 Medida Provisória n ${ }^{\circ}$ 2.158-35, de 24 de agosto de 2001

2.2 Instrução Normativa $\mathrm{n}^{\circ} 265$, de 20 de dezembro de 2002

2.3 Ato Declaratório Executivo Cofis $n^{\circ} 20$, de $1^{\circ}$ de outubro de 200330

2.4 Ato Declaratório Executivo Cofis $n^{\circ} 7$, de 20 de maio de 2004

2.5 Ato Declaratório Executivo Cofis $n^{\circ} 8$, de 9 de julho de 2004

2.6 Ato Declaratório Executivo Cofis $n^{\circ}$ 9, de 20 de julho de 2004

2.7 Ato Declaratório Executivo Cofis $n^{0} 13$, de 7 de dezembro de 200435

2.8 Ato Declaratório Executivo Cofis $n^{\circ}$ 9, de 13 de abril de 2005

2.9 Participação do Inmetro 36

2.10 Breve avaliação econômica do impacto da Medida Provisória 41

Capítulo 3 Métodos de diferenciação de bebidas e Incerteza de medição44

3.1 Densimetria 44

3.2 Potenciometria $\quad 45$

3.3 Condutividade 47

3.4 Índice de refração com a utilização da fibra óptica 50

3.5 Incerteza de Medição 56

Capítulo 4 Medições (laboratoriais X processo) e Cálculos 59

4.1 Densidade absoluta: resultados obtidos nos laboratórios das

Unidades e na linha de produção da AmBev 59

$4.2 \mathrm{pH}$ (potenciometria): resultados obtidos no Laboratório Central da AmBev

4.3 Condutividade: resultados obtidos no Laboratório Central e na 
linha de produção da AmBev $\quad 71$

4.4 Índice de refração: resultados obtidos no LSFO da PUC-Rio $\quad 81$

4.4.1 Refratômetro com fibra monomodo 82

4.4.2 Refratômetro com fibra multimodo 93

4.5 Cálculo da Incerteza 109

4.6 Discussão dos resultados e sugestão da melhor metodologia 118

Capítulo 5 Conclusões e recomendações 120

Capítulo 6 Referências bibliográficas 122

ANEXOS 127

ANEXO A MP N ${ }^{\circ}$ 2.158-35, de 24 de agosto de 2001

ANEXO B Instrução Normativa $N^{\circ}$ 265, de 20 dezembro de 2002

ANEXO C ADE COFIS $\mathrm{N}^{\circ} 20$, de $1^{\circ}$ de outubro de 2003

ANEXO D ADE COFIS N ${ }^{\circ}$ 7, de 20 de maio de 2004

ANEXO E ADE COFIS N ${ }^{\circ}$ 8, de 9 de julho de 2004

ANEXO F ADE COFIS N 9 , de 20 de julho de 2004

ANEXO G ADE COFIS Nº 13, de 7 de dezembro de 2004

ANEXO H ADE COFIS N 9 , de 13 de abril de 2005 


\section{Lista de figuras}

Figura 1 - Aspectos envolvidos em cada estágio da cadeia produtiva de bebidas que poderiam ser utilizados para o controle de impostos. A medição do volume produzido durante o processo produtivo é a forma mais eficiente para controle de impostos. [3] 23

Figura 2 - Características do Sistema de Medição de Vazão (SMV). [3] 24

Figura 3 - Montagem do Protótipo do SMV na linha de enchimento da Filial Jaguariúna (AmBev). O número 1 identifica o medidor de vazão, o número 2 o condutivímetro, e o número 3 o cabeamento de transmissão de dados. [3]

Figura 4 - Exemplos internacionais de uso do Sistema de Medição de Vazão. [3]

Figura 5 - Vista panorâmica do packaging, linha de envase de lata da Filial Rio de Janeiro (AmBev).

Figura 6 - Vista da cúpula da enchedora de latas da Filial Rio de Janeiro (AmBev).

Figura 7 - Visão interna da cúpula da enchedora onde estão instalados, devidamente lacrados dentro do gabinete de metal (invólucro físico lacrado indicado pela seta vermelha), o medidor de vazão, o condutivímetro e o medidor de temperatura.

Figura 8 - Painel de visualização de todos os parâmetros lidos, cujo acesso está devidamente lacrado (lacre acima, à esquerda, indicado pela seta vermelha).

Figura 10 - Distribuição da incidência de tributos até a chegada do produto ao consumidor final. [45]

Figura 11 - Princípio do funcionamento do Condutivímetro utilizado no SMV. [9]

Figura 12 - Campos estacionários devidos a fontes fixas: magnético e elétrico. [48]

Figura 13 - A fibra óptica pode ser comparada, em suas dimensões, a um fio de cabelo. [29] 
Figura 14 - Fibras ópticas. A luz quando aplicada a uma das extremidades, após percorrer a fibra, sai pela outra extremidade. [29]

Figura 15 - Visualização das várias camadas do cabo de fibra óptica, da esquerda para a direita: capa protetora externa (plástico), capa protetora interna (plástico), casca (camada em torno do núcleo que impede a saída da luz do núcleo) e o núcleo. [29]

Figura 16 - A reflexão interna total na fibra óptica entre o núcleo $\left(n_{1}\right)$ e a casca $\left(\mathrm{n}_{2}\right)$ ocorre para ângulos de incidência maiores que o ângulo crítico. (a) Para ângulos de incidência menores que o ângulo crítico, a refração ainda ocorre. (b) Feixe incidindo no ângulo crítico, ângulo no qual o ângulo de refração é igual a $90^{\circ}$. [50]

Figura 17 - Sistema óptico básico. [49] 55

Figura 18: Tipos de Bebidas $X$ Densidade Absoluta, resultados obtidos em alguns laboratórios de Unidades AmBev

Figura 19 - Valores de $\mathrm{pH}$ em função da temperatura de alguns Refrigenancs (refrigerantes e não-alcoólicos como chás).

69

Figura 20 - Valores de pH em função da temperatura de algumas Cervejas. 69

Figura 21 - Valores de pH em função da temperatura para diversos tipos de bebidas.

Figura 22 - Medidas de Temperatura X Condutividade de diversos tipos de bebidas.

Figura 23 - Medidas de Tempo X Condutividade de diversos tipos de bebidas na Linha de Envase. (Dados conseguidos através do condutivímetro que faz parte do SMV já instalado e devidamente homologado na linha de envase de latas da Filial Rio de Janeiro (AmBev).

Figura 24 - Medições do Índice de Refração em cervejas com e sem álcool (Liber), obtidos com um sensor à fibra óptica monomodo fabricado pela Gavea Sensors (medições realizadas no LSFO da PUC-Rio).

Figura 25 - Medições do Índice de Refração em refrigerantes do tipo comum, obtidos com um sensor à fibra óptica monomodo fabricado pela Gavea Sensors (medições realizadas no LSFO da PUC-Rio).

Figura 26 - Medições do Índice de Refração em refrigerantes do tipo diet, obtidos com um sensor à fibra óptica monomodo fabricado pela Gavea Sensors (medições realizadas no LSFO da PUC-Rio).

Figura 28 - Medições do Índice de Refração para todas as bebidas testadas, obtidos com um sensor à fibra óptica monomodo fabricado pela Gavea 
Sensors (medições realizadas no LSFO da PUC-Rio).

Figura 30 - Montagem realizada para a execução das análises com a utilização da fibra óptica do tipo multimodo, fabricado pela Gavea Sensors (medições realizadas no LSFO da PUC-Rio).

Figura 31 - Medições do Índice de Refração para cervejas com e sem álcool, obtidos no laboratório da PUC-Rio, com a utilização da fibra óptica do tipo multimodo.

Figura 32 - Medições do Índice de Refração para refrigerantes do tipo comum, obtidos com um sensor à fibra óptica multimodo fabricado pela Gavea Sensors (medições realizadas no LSFO da PUC-Rio).

Figura 33 - Medições do Índice de Refração para refrigerantes do tipo light, obtidos com um sensor à fibra óptica multimodo fabricado pela Gavea Sensors (medições realizadas no LSFO da PUC-Rio).

100

Figura 34 - Medições do Índice de Refração para chás, obtidos com um sensor à fibra óptica multimodo fabricado pela Gavea Sensors (medições realizadas no LSFO da PUC-Rio).

100

Figura 35 - Medições do Índice de Refração para todas as bebidas testadas, obtidos com um sensor à fibra óptica multimodo fabricado pela Gavea Sensors (medições realizadas no LSFO da PUC-Rio).

Figura 36 - Banho para acondicionamento das amostras composto de gelo moído e água gelada com temperatura entre 0 e $8^{\circ} \mathrm{C}$. Observa-se também a fibra do tipo multimodo e o PT100 utilizados para a realização das medidas devidamente inseridos na bebida (amostra).

105

Figura 37 - Refratômetro utilizado para as medições, no qual a fibra óptica do tipo multimodo estava conectada (cabo amarelo).

105

Figura 38 - Controlador de temperatura no qual o PT100 estava conectado, utilizado para as medições com a fibra do tipo multimodo.

106

Figura 39 - Placa de aquisição utilizada para as medições com a fibra do tipo multimodo.

106

Figura 40 - Amostras utilizadas para as medições com a fibra do tipo multimodo.

107

Figura 41 - Sistema LabVIEW versão 6.1 com programa DOF, utilizados para as medições com a fibra do tipo multimodo.

107

Figura 43 - Combinação dos dados dos sistemas "Medição de Condutividade" e "Refratômetro Óptico" para diferentes bebidas.

108

Figura 44 - Medições do Tempo X Índice de Refração para Skol (latas iguais), 
obtidos com um sensor à fibra óptica multimodo fabricado pela Gavea Sensors (medições realizadas no LSFO da PUC-Rio) para o cálculo da Repetitividade do método. 116

Figura 45 - Medições do Tempo X Índice de Refração para Skol (latas diferentes), obtidos com um sensor à fibra óptica multimodo fabricado pela Gavea Sensors (medições realizadas no LSFO da PUC-Rio) para o cálculo da Reprodutibilidade do método. 


\section{Lista de tabelas}

Tabela 1: Resultados de densidade absoluta $\left(\mathrm{g} / \mathrm{cm}^{3}\right)$ obtidos por laboratórios das Unidades AmBev

Tabela 2: Densidades Absolutas medidas nos laboratórios comparados às obtidas pelo protótipo instalado em linha de envase. 63

Tabela 3: Resultados obtidos através do protótipo do densímetro instalado na linha de envase da Fábrica Águas Claras do Sul (Viamão) da AmBev. Observa-se que durante o processo de CIP, a densidade absoluta fica muito próxima à faixa dos refrigerantes diet/light. .64

Tabela 4: Resultados de $\mathrm{pH}$ em sete (7) refrigenancs. Análises realizadas no Laboratório Central da AmBev. 67

Tabela 5: Resultados de pH em quatro (4) cervejas alcoólicas e uma (1) cerveja não alcoólica. Análises realizadas no Laboratório Central da AmBev. 68

Tabela 6: Resultados de condutividade em refrigenancs. Análises realizadas no Laboratório Central da AmBev .73

Tabela 7: Resultados de condutividade em cervejas. Análises realizadas no Laboratório Central da AmBev. 74

Tabela 8: Resultados de condutividade em Linha de diversos tipos de bebidas. Dados obtidos durante o envase na Linha de latas da Filial Rio de Janeiro.

Tabela 9 - Dados obtidos para cervejas com e sem álcool com a utilização de um sensor à fibra óptica do tipo monomodo fabricado pela Gavea Sensors (medições realizadas no LSFO da PUC-Rio).

Tabela 10 - Dados obtidos para refrigerantes do tipo comum com a utilização de um sensor à fibra óptica do tipo monomodo fabricado pela Gavea Sensors (medições realizadas no LSFO da PUC-Rio). 84

Tabela 11 - Dados obtidos para refrigerantes do tipo diet com a utilização de um sensor à fibra óptica do tipo monomodo fabricado pela Gavea Sensors (medições realizadas no LSFO da PUC-Rio).

Tabela 12 - Dados obtidos para chás com a utilização de um sensor à fibra óptica do tipo monomodo fabricado pela Gavea Sensors (medições realizadas no LSFO da PUC-Rio).

Tabela 13 - Exemplo da aplicação do Teste de Dixon nos dados obtidos com a 
utilização do sensor à fibra monomodo fabricado pela Gavea Sensors (medições realizadas no LSFO da PUC-Rio). ............................................ 91

Tabela 14 - Observação do efeito da temperatura nos dados obtidos com a utilização do sensor à fibra monomodo fabricado pela Gavea Sensors (medições realizadas no LSFO da PUC-Rio).

Tabela 15 - Dados obtidos para cervejas com e sem álcool com a utilização de um sensor à fibra óptica multimodo fabricado pela Gavea Sensors (medições realizadas no LSFO da PUC-Rio). 95

Tabela 16 - Dados obtidos para refrigerantes do tipo diet ou light com a utilização de um sensor à fibra óptica multimodo fabricado pela Gavea Sensors (medições realizadas no LSFO da PUC-Rio). .96

Tabela 17 - Dados obtidos para refrigerantes do tipo comum com a utilização de um sensor à fibra óptica multimodo fabricado pela Gavea Sensors (medições realizadas no LSFO da PUC-Rio).

Tabela 18 - Dados obtidos para chás com a utilização de um sensor à fibra óptica multimodo fabricado pela Gavea Sensors (medições realizadas no LSFO da PUC-Rio). 98

Tabela 19 - Comparativo entre os dados de índice de refração obtidos com a utilização dos sensores a fibra óptica monomodo e a fibra óptica multimodo, fabricados pela Gavea Sensors (medições realizadas no LSFO da PUCRio). 103

Tabela 20: Cálculo da Incerteza do Tipo A dos resultados de condutividade obtidos na linha de envase. 110

Tabela 21: Cálculo da Incerteza do Tipo A dos resultados de índice de refração com fibra monomodo.

Tabela 22: Cálculo da Incerteza do Tipo A dos resultados de índice de refração com fibra multimodo. 112

Tabela 23: Cálculo da Incerteza do Tipo B dos valores de condutividade obtidos em laboratório.

Tabela 24 - Dados obtidos com a utilização de um sensor à fibra óptica multimodo fabricado pela Gavea Sensors (medições realizadas no LSFO da PUC-Rio), da Skol 114 para o cálculo da Repetitividade do método 114

Tabela 25 - Dados obtidos com a utilização de um sensor à fibra óptica multimodo fabricado pela Gavea Sensors (medições realizadas no LSFO da PUC-Rio), da Skol 
para o cálculo da Reprodutibilidade do método.

Tabela 26 - Cálculo da Incerteza do Tipo A, considerado como Incerteza do Tipo $\mathrm{B}$,

aplicado à repetitividade do índice de refração com a utilização de um sensor à fibra óptica multimodo ........................................................................117

Tabela 27 - Cálculo da Incerteza do Tipo A, considerado como Incerteza do Tipo $\mathrm{B}$, 117 aplicado à reprodutibilidade do índice de refração com a utilização de um sensor à fibra óptica multimodo 


\title{
Lista de Abreviaturas e Símbolos
}

\author{
ADE - Ato Declaratório Executivo \\ AmBev - American Beverage Company \\ ASTM - American Society for Testing and Materials \\ CenPRA - Centro de Pesquisas Renato Archer \\ CIP - Cleanning in Place
}

Cofins - Contribuição para Financiamento de Seguridade Social

COFIS - Coordenação Geral do Sistema de Fiscalização

d.d.p. - Diferença de Potencial

DOF - Detector Óptico de Fluidos

DOU - Diário Oficial da União

EMI - Electro Magnetic Interference

FDA - Food and Drugs Administration

FINEP - Financiadora de Estudos e Projetos

FNDCT - Fundo Nacional de Desenvolvimento Científico e Tecnológico

ICMS - Imposto sobre Circulação de Mercadorias

Inmetro - Instituto Nacional de Metrologia, Normalização e Qualidade Industrial

IPI - Imposto sobre Produtos Industrializados

LED - Light-Emitting Diode

LSFO - Laboratório de Sensores a Fibra Óptica

MCT - Ministério da Ciência e Tecnologia

MP - Medida Provisória

NIST - National Institute of Standard and Technology - USA 
OIML - Organização Internacional de Metrologia Legal

PIS - Programa de Integração Social

PósMQI - Programa de Pós-Graduação em Metrologia para Qualidade e Inovação

PUC-Rio - Pontifícia Universidade Católica do Rio de Janeiro

Refrigenancs - Refrigerantes e não alcoólicos

SI - Sistema Internacional de Unidades

Sindicerv - Sindicato Nacional da Indústria da Cerveja

SMV - Sistema de Medição de Vazão

STP - Standard Temperature and Pressure

TIB - Tecnologia Industrial Básica

TIPI - Tabela de Incidência do Imposto sobre Produtos Industrializados

VIM - Vocabulário Internacional de Metrologia 